\title{
A Brief Analysis of the Interactive Teaching Methods and Functions in Teaching Chinese as a Foreign Language
}

\author{
Zhu Hongzhi ${ }^{1, \text { a }}$, Li Yingbo*1
}

\begin{abstract}
${ }^{1}$ School of Humanities and Management, Shaanxi University of Chinese Medicine xianyang, Shaanxi, China 1340610159@qq.com
\end{abstract}

\begin{abstract}
with the improvement of China's comprehensive national strength in teaching Chinese as a foreign language has been booming, many kinds of teaching method to get the different progress and development, the interactive teaching is more and more get people's attention and concern, from the teachers, students, teaching materials, three angles to illustrate in interactive teaching methods and role in teaching Chinese as a foreign language, through this article to let teachers in the teaching process to pay more attention on the diversity of teaching methods.
\end{abstract}

Keywords: classroom teaching, interactive teaching, second language teaching

\section{浅析对外汉语教学中互动教学的方法及作用

\author{
朱宏治 1 , a 李莹波*1
}

${ }^{1}$ 陕西中医药大学人文管理学院 咸阳, 陕西, 中国

a1340610159@qq.com

*通讯作者: 1376564600@qq.com

摘要

随着我国综合国力的提高我国对外汉语教学事业得到了蓬勃发展, 多种教法学法得到了不同的进步与发展, 其 中互动教学越来越得到人们的重视和关注, 本文从教师, 学生, 教材, 三个角度出发来阐述在对外汉语教学中 互动教学的方法及作用, 旨在通过本文让教师在教学过程中对教学方法的多样性给予更多关注。

关键词: 课堂教学, 互动教学, 二语教学

\section{1. 互动教学的含义, 特性及影响}

\section{1. 含义}

互动一词对于我们来说并不陌生, 它存在于我们 生活的各个领域, 作为一种教学方法, 学习方法, 它 贯穿于教与学过程的始终。包括师生之间, 生生之间, 既包括语言互动, 又包括非语言互动。互动教学把教 学过程看成是一个有机整体, 在这一过程中为了更好 地达到教学目标, 所采取的学习策略与教学策略。

\section{2. 特性}

（1）互动教学具有计划性和目的性
互动教学是为教学目标所服务, 不同于一般的人 际互动, 在课堂教学中采取何种互动方式, 应视教学 内容, 学生主体情况, 学习状态等情况而定。这些既 可以在教师备课过程中做出细节预设，同时也可以根 据实际教学情形临时增设删减。教学互动从教师角度 而言属于教学策略范畴, 短期目的在于课堂目标的高 效完成, 可以更好的传递知识, 让学生更好的理解吸 收。最终目的是促进学生综合全面的发展。

\section{（2）互动教学具有主动性和系统性}

互动强调的是主体之间的主动交流与分享, 共同 营造和谐愉快的氛围, 因此这一互动不能流于形式, 或是漫无目的的信息交流。应充分调动师生参与的积 极性和主动性。主动参与的互动才会具有融入感与体 验感, 因此也才能更好的达到我们的教学目标。同时 
课程互动是连续的, 渐进的, 不是中断的, 跳跃的。 互动应贯穿教学过程的始终。将互动教学作为教学方 法, 复习方法, 练习方法等等, 同时互动要求循序渐 进, 在于学生建立良好的印象与信任的基础上才能事 半功倍。不同的学科均可以视本学科的实际情况选择 合适的互动方法策略。

\section{（3）互动教学具有继承性与创新性}

互动教学由来已久, 归根到底就是教学主体客体 之间的双向交流，这一实践操作具有悠久的历史，人 们总是在自觉或不自觉中加以运用发展, 同时互动教 学又具有与时俱进的特点, 在传统的师生问答的基础 上, 逐渐发展出生生交流, 家校互动等多种形式, 尤 其是随着多媒体技术的日益发展, 体验式教学给互动 创造了更多的空间和可能性。各种教学游戏, 教学互 动软件, 在寓教于乐中将知识与技能传递给学生, 提 升教学的趣味性与多样性。

\section{3. 作用}

\subsection{1. 对学习者而言}

(1) 有利于提高学生开口率, 增加可理解性输入

根据互动假设理论和输出假设理论, 语言的学习 是在外部的客观环境与内在的学习机制共同影响下 进行的, 课堂互动中无论是生生互动还是师生互动, 都提供了大量丰富的语料, 提供了可理解性输入与语 言输出的机会, 学习者通过留意不同的语言形式与意 义, 注意到不同的语言表达方式与偏误, 从而可以更 好地验证自己的假设, 降低语言偪化出现的概率, 加 快学习者中介语向目的语过渡的进程。

\section{（2）有利于提高学生的自信心与表达能力}

互动教学努力的营造积极愉悦的学习氛围, 学生 一方面是倾听者另一方面是表达者。在倾听过程中, 学习者也在努力的做出相应的回应与反馈, 这就为接 下来的语言表达提出了更高的要求, 当自身的语言能 力无法满足所要表达内容时, 学习者可以采取多种学 习策略, 例如小组学习探讨, 咨询老师, 同义替代相 应词语, 使用肢体动作等等, 这些都有利于增强学生 的自信心与表达能力, 从而更好的进行语言学习。

\section{（3）有助于提升学生思辨能力提高全局意识}

互动教学中要求师生双方即时性互动, 因此双方 必须保持着较高的注意力, 同时能捕捉到双方交流信 息中的核心部分并进行有效回答。因此此种教学方法 可以有效的提升学生的思辨能力, 理解能力, 班级授 课制中, 教师要面对的是全体学生, 因此学生集体之 间的互动尤为重要, 在与同学互动之中承担着怎样的 角色, 处于怎样的位置, 有赖于学生之间的沟通配合, 因此可以有限的提升学生的大局意识与整体观念。

\subsection{2. 对教师而言}

(1) 有助于教学活动的顺利展开, 提高学生学习 效率

二语教学的目的是让学习者学会使用二语进行 交际, 互动教学营造轻松愉快氛围, 有利于打破师生 之间由于文化差异等诸多因素带来的陌生与隔阂, 因 此有助于教师顺利的进行课堂教学。学生积极主动的 与老师同学进行交流分享, 对初学者而言有利于更好 的度过文化震荡期, 对中高级学习者而言互动教学可 以加深对二语文化的理解与感悟。从而提升学生的学 习效率与效果。同时可以加强师生之间相互信任, 营 造积极向上的班集体氛围, 良好的班集体需要师生共 同建设, 对教师而言同样是教学工作的一部分, 优秀 的教师不单单传授学生知识, 更能为学生的全面发展 提供助力。

\section{（2）更好的了解学生进行针对性教学}

班级授课制目前仍是主流的教学方式, 它可以在 有限的时间空间内进行大规模教学有助于学生的培 养, 但同时对学生的个性关注度不够, 对学生的个性 全面发展造成一定的影响, 互动教学强调高效个性化 特点, 通过对同一内容的不同回答, 可以发现学生们 关注事物的角度差异, 理解学生认知的不同, 互动交 流可以更好的发现学生的个性化特征, 进行有针对性 地教学, 提高教学效果, 促进学生在综合全面的发展。

（3）促进教师专业化发展, 打造精品课程

互动教学是新手教师发展成为专家型教师必不 可少的教学技能, 新手教师在从教之初往往忽视互动 教学, 更多的是满堂灌的教学方式, 因此造成教师与 学生彼此之间出现困惑, 互动教学可以帮助教师迅速 的成长, 更好的了解学生, 有助于教师更加自信的处 理课堂上的突发事件，同时提升与家长之间的沟通与 配合, 更好的形成教学合力。另一方面能熟练的运用 互动教学策略, 有助于打造精品课程, 将课堂专业化, 精品化。

\section{2. 教师如何更好的运用互动教学策略}

\section{1. 恰当地运用多种教学方法}

(1) 教无定法, 教学方法随着教学内容, 教学对 象的不同而有所差别, 因此教师在教学的过程中应综 合运用多种教学方法, 例如全身反应法, 游戏法等等 可以有效的调动学生多种感官同时协调运作, 增强学 习效果, 但此时教师应注意在运用教学方法时要具有 差异性, 面对不同的学生个体互动应有所差别, 做到 因材施教。同时教学方法的多样性是教师教学艺术的 体现, 是新手教师向成熟的重要标志。

（2）善于倾听总结, 创新互动教学方法。新手教 师除了在自己的课堂教学中汲取信息, 同时也应善于 倾听总结, 向成手教师请教同时对其上课过程进行观 
摩，学习方法技巧帮助自己快速的成长，同时结合自 身的风格优势, 对自己的教学方法进行优化升级, 以 期达到更加自由妚熟的目标。

\section{2. 创设多种教学情境, 善于利用教辅资源}

(1) 互动教学贯穿于教学过程的全过程, 并不仅 仅局限于课中，课前的引入，课后的复习巩固均可采 用此种教学方式，同时互动并非意味着仅我问你答， 教师可以发散思维，例如教师可以通过故事引入创设 故事情境, 从而推动学生参与热情的提高, 也可以通 过课前演讲, 课后辩论, 来加强师生之间的互动交流, 从而教师也可以更好的检验学生的学习效果。近年来 教师创造性的将话剧, 舞台剧, 舞蹈等多种形式运用 于互动教学之中取得了意想不到的教学效果值得我 们借鉴和思考。

（2）随着教学技术的迅速发展, 人工智能, 计算 机等相关科学技术应用于教学之中, 推动教学智能化, 信息化发展。多媒体技术集视听说多种感官刺激于一 体, 充分调动学生的学习热情, 多媒体技术优势在于: 所涵盖信息量广泛全面, 同时以趣味性的方式呈现出 来易于学生接受.但同时教师应辩证的看待多媒体技 术, 多媒体技术在一定程度上解放了教师压力, 但教 师不可过分依赖, 导致传统的教学方式缺失。第二, 多媒体技术尚有诸多发展空间, 例如在如何加强学生 与多媒体互动方面, 集中学生注意力方面存在改进完 善空间。第三: 多媒体的运用要因课程类型, 教学内 容等等的不同进行选择, 并非课堂必须运用, 教学方 法手段各有所长不可一概而论。

(3) 将互动教学融于实践之中, 实践是检验真理 的唯一标准, 二语课程学习具有一定的难度, 因此采 用走出去的实践方法能更好的激发学生的学习兴趣, 同时加深对知识技能的理解, 互动教学不局限与三尺 讲台, 也不局限于面对面交流。师生关系也可以是朋 友关系, 积极组织并参加实践活动, 可以加深师生之 间的理解, 同时互动又不仅仅局限于当面交流互动, 我们也可以组织线上的互动交流，更加的便捷与高效。

\section{3. 学生如何利用互动学习实现学习目标}

\section{1. 加强主动参与意识, 勇于融入表达}

(1) 汉语属印欧语系语言, 在汉语的学习过程 中, 二语学习者普遍认为语音方面声调是最难以习得 的, 因此学生在学习语音时往往难以开口, 因此导致 学习进程止步不前, 甚至中高级阶段学习者也出现不 同程度的语音偏误和语音僵化现象。因此在学习的过 程中, 学习者应主动积极参与课堂互动, 利用师生互 动的方式可以更好的习得目的语, 同时师生互动, 生 生互动为学生提供浓厚的语言氛围, 促进二语学习者 二语技能的形成与发展, 良好愉悦的氛围有助于学生
更好的适应跨文化环境，更加充分自信的融入目的语 社团。

（2）善于反思总结提升自主学习能力。互动教 学能带给师生的不仅仅是愉悦的课堂氛围, 也不仅仅 是单纯为了实现教学目标, 同时它也带给了学生更多 的思考与想象, 学生可以通过与教师同学之间的互动, 更好的提升自己的语言组织能力, 也能通过互动加深 自己对知识的全面理解以及对问题的看法及观点, 这 对学生长远的发展尤为重要, 从互动中总结的经验得 到的灵感可以为学生接下来的发展注入更多的可能 性。

\section{4. 教辅资料的选择与利用}

\section{1. 教辅资料的选择}

教辅资料在教学过程中起着纽带的作用, 它是教 学内容的物质承担者, 因此选择合适的教辅资料尤为 重要, 好的教辅资料可以提高教师教学效果, 提升学 生学习质量, 因此在选择教辅资料时应注意以下几点:

（1）教材选择应具有针对性和差异性

对外汉语教学不同于国内汉语教学, 二者学习主 体存在诸多差异, 因此在教材的选择上为了更好的适 应学习者的实际情况, 便于教师开展教学活动, 教材 选择应具有针对性, 适应学生基础, 符合学生生理心 理发展特点, 服务于教学目标, 才能更好的达到教学 目的。互动教学要求教学双方大致处于相同的认知范 围, 如果教材难度过大, 或者与本土化教材相差甚远, 学生就会产生畏难情绪, 从而不愿意参与课堂活动, 导致教学效果大打折扣。

\section{（2）教辅资料的选择应尊重参考学生的意愿}

这点在中高级教学中值得老师们思考注意, 经过 一段时间的学习, 学习者的基础出现较大差别, 另外 由于学习目的, 学习时间的不同, 学习者对二语学习 所呈现的状态也是千差万别, 因此在教辅资料的选择 上应考虑学生的诉求与想法, 在互动教学之中, 应结 合学生实际情况及需求进行调整与改变, 必要时可以 对教辅资料进行合理的替换与修改。

\section{2. 如何高效利用教辅资源}

(1) 课前教师应对教材内容进行评估设计, 评估 教材难易程度, 学生的易混易错点, 设计相应教学方 案, 合理的分配讲练时间, 同时要求学生进行相应预 习, 并适当的进行教学引导激发学生期待值提升学生 学习兴趣, 同时带着任务进行预习可以更好的让学生 把握重难点。

（2）课中教师应结合教学实际情况对学生在教 学中呈现的亮点及问题进行梳理强化, 保证教学重点 可以很好的被学生吸收和理解。课后布置相应作业任 务, 强化课后学生自主学习自主实践能力。 
（3）教学资源是教学目标实现的重要推动因素, 因此鼓励有条件的地区制定符合本地区学情的有针 对性地教辅资料, 内容涵盖理论与实践双重部分, 应 紧扣学科前沿理念及最新素材, 同时教辅资料的设计 具有趣味性充分调动学生学习的积极性, 起到事半功 倍的理想效果。

（4）课堂教学的主体是老师, 因此我们在利用教 辅资源时候应有所侧重, 教学活动是情感交流, 师生 增进感情的过程, 在这一过程中教师的言传身教会对 学生的全面发展产生重要影响, 因此多多的与学生进 行分享沟通, 可以将自己的实践经历作为案例与学生 交流分享, 这样才能达到教学相长的和谐效果。

\section{（5）鼓励有条件地区开展校本课程资源}

校本课程是学校个性化的集中体现, 每所学校都 有自己的发展历史, 都有自己关于教书育人理念的理 解, 同时校本课程是一项重要的课程资源, 学校可以 开发校本教辅资源, 一方面校本资源体现本土化特征, 与学生, 学生有更多的契合之处, 可以更好的让学生 理解课堂知识同时又能很好的加以运用, 另一方面一 方水土，一方文化。校本教辅资源的开发是一个长期 的过程, 因此充分的利用校本资源有助于地域文化的 传承。

\section{5. 结论}

教学是一项艺术性的创作过程, 在这一过程中教 师与学生均是课堂的主人, 因此充分调动二者参与热 情不但有利于构建新型师生关系, 同时能够推动教学 活动健康有序进行, 对外汉语教学有其自身的独特性, 由于师生所处的文化教育背景的不同, 在教学中难免 出现困惑与不解, 对此师生之间应相互理解求同存异。 教学互动更多的要围绕着教学内容展开, 谨慎讨论敏 感话题, 避免引起不要的冲突与矛盾。互动教学是一 种行之有效的教学方式, 在实际运用过程中一定要结 合实际的教育教学情况, 学生的生理心理特点灵活的 进行选择处理。

\section{REFERENCES}

[1] Chen Lp (2005) The connotation, level and evolution of interactive effect in classroom teaching. Journal of Guangxi Education Sixth issue: $34-37$

[2] Du X (2006) Aspects of the Teaching mode of Oral Chinese as a Foreign Language. Language application second phase:152-154

[3] Fu Wf 2007.A comparative study of learner anxiety and learner participation in five Kinds of Chinese Classroom Interaction activities, Master's thesis, East China Normal University

[4] Zhang linjun 2002. teacher-student interaction and development of learners' language communicative competence, Beijing language and culture university master's thesis

[5] [5] Wang Jianqiang. 2011 Application of Interactive Teaching in Teaching Chinese as a Foreign Language, Master's thesis of Nanjing Normal University 